\title{
Ein Update zur Onlineplattform «Guidelines Schweiz»
}

\author{
Stefanie Hostettlera, Esther Kraft ${ }^{\mathrm{b}}$, Christoph Bosshard ${ }^{\mathrm{c}}$

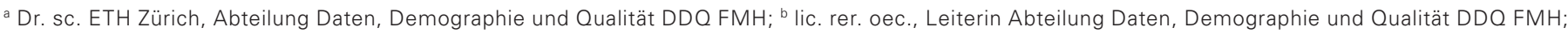 \\ c Dr. med., Vizepräsident der FMH, Departementsverantwortlicher Daten, Demographie und Qualität DDQ FMH
}

Korrespondenz: FMH/Abteilung DDQ

Elfenstrasse 18

CH-3000 Bern 15

Tel. 0313591111

ddq[at]fmh.ch
Medizinische Guidelines sind systematisch entwickelte Aussagen, die helfen sollen, im Interesse der bestmöglichen Behandlung der Patientinnen und $\mathrm{Pa}$ tienten evidenzbasierte Entscheidungen zu treffen. Es gibt bereits unzählige Guidelines und laufend entstehen neue. Dies erschwert den Überblick und führt zu Unsicherheiten bei deren Verwendung. Der Einschluss qualitativ mangelhafter Studien, Nicht-Publikation von Studien (Publication bias), Interessenkonflikten etc. können zu widersprüchlichen Guidelines oder Verzerrungen führen. Zudem gelten Guidelines in der Regel für einzelne Krankheitsbilder. In der medizinischen Praxis stellt jedoch der Umgang mit Guidelines bei Multimorbidität eine Herausforderung dar.

Um einen Überblick über die unzähligen Guidelines zu schaffen und Unsicherheiten hinsichtlich der Validität der verwendeten Guidelines zu reduzieren, hat die Schweizerische Akademie der Qualität in der Medizin SAQM 2017 die Onlineplattform «Guidelines Schweiz» realisiert (https://guidelines.fmh.ch). Die Plattform soll als Orientierungs- und Vernetzungshilfe für Interessierte dienen.

Guidelines sind in einheitlichen Strukturen abrufbar und enthalten Angaben zu den Anwendern, zur Zielgruppe, zu den relevanten Fachgebieten, zur Entwicklung etc. Der Inhalt der Guidelines bzw. die eigentliche Handlungsanweisung kann mit Dokumenten und/ oder Websites verlinkt werden. Die dokumentierten Guidelines erfüllen definierte Mindestkriterien. Beispielsweise sind die Information zur Finanzierung offengelegt, die Interessenbindungen der GuidelineKommissionsmitglieder dokumentiert oder die Gültigkeitsdauer der Guideline bekannt. Mit dieser Massnahme werden qualitativ gute Guidelines gefördert.

Im Weiteren soll die Onlineplattfom «Guidelines Schweiz» folgende Bereiche fördern:

- Kritische Auseinandersetzung mit der Qualität von Guidelines

- Einheitliche Anwendung von Guidelines innerhalb eines medizinischen Fachgebietes

- Interdisziplinärer und interprofessioneller Austausch

- Diskurs zur Anwendung von Guidelines bei Multimorbidität.

\section{Aufnahme von neuen Guidelines}

Nach wie vor gibt es medizinische Guidelines, welche noch nicht auf der Plattform präsent sind und einem breiten Fachpublikum vorgestellt werden sollten. Um Fachgesellschaften, Spitälern/Kliniken und weiteren Organisationen im Gesundheitswesen die Möglichkeit zu bieten, weitere von ihnen anerkannte Guidelines zu dokumentieren, führt die FMH aktuell ein Update durch. So wird transparent gemacht, wer welche Guidelines zur Anwendung empfiehlt.

\section{Teilnahme an der Onlineplattform}

Verfügt Ihre Organisation über Guidelines und möchte diese dokumentieren?

Um zum Fragebogen zu gelangen, klicken Sie bitte auf folgenden Link:

Onlinefragebogen für Guidelines (http://www.onlineumfragen.com/login.cfm?umfrage=77323). Oder melden Sie sich per E-Mail oder telefonisch bei der Schweizerischen Akademie für Qualität in der Medizin SAQM (saqm[at]fmh.ch oder 0313591111 ).

Mit derTeilnahme am Projekt unterstützen Sie qualitativ hochstehende Guidelines und tragen aktiv zur Qualitätsverbesserung bei. Vielen Dank für Ihre Mitarbeit!
Mit Hilfe eines Onlinefragebogens werden die Angaben zu den Guidelines erfasst. Die Dokumentation pro Guideline nimmt nur wenige Minuten in Anspruch. Die elektronische Befragung startet am 10. und läuft bis am 31. Oktober 2018. Ende November 2018 wird die Plattform online geschaltet. Fortan wird die Befragung einmal jährlich durchgeführt und mit neuen Guidelines ergänzt und aktualisiert. 